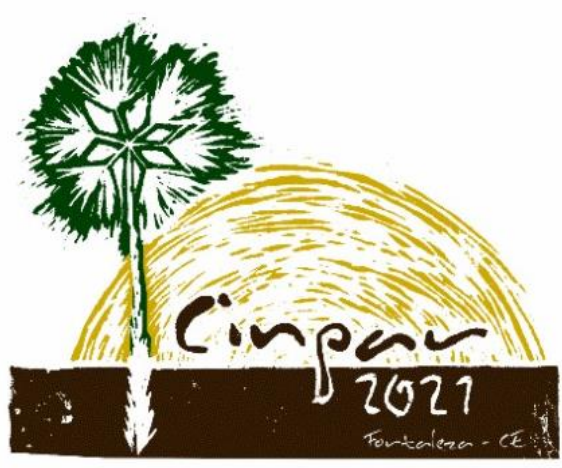

XVII Congresso Internacional sobre Patologia e

Reabilitação das Construções

XVII Congreso Internacional sobre Patología y Rehabilitación de las Construcciones

XVII International Conference on Pathology and Constructions Rehabilitation

FORTALEZA (Brasil), 3 a 5 de junho de 2021

https://doi.org/10.4322/CINPAR.2021.094

\title{
Vulnerabilidad sísmica de edificios escolares: de la evaluación al refuerzo
}

\author{
Victor ROLDAN ${ }^{1}$, Gustavo PALAZZO², Marcelo GUZMAN ${ }^{3}$ \\ ${ }^{1}$ Universidad Tecnológica Nacional, FRM, Ceredetec, Argentina, victor roldan890@hotmail.com \\ 2 Universidad Tecnológica Nacional, FRM, Ceredetec, gpalazzo@frm.utn.edu.ar \\ 3 Universidad Tecnológica Nacional, FRM, Ceredetec, Argentina, mguzman@frm.utn.edu.ar
}

Resumen: En zonas sísmicas existen muchas construcciones diseñadas y construidas con reglamentos antiguos o sin ellos, las cuales podrían ser vulnerables frente a terremotos destructivos. En este trabajo se consideran en especial los edificios escolares, dada la importante función que cumplen. Para estas construcciones consideradas en forma individual es necesario contar con un protocolo para conocer su nivel de vulnerabilidad y, si fuera necesario, estrategias admisibles de refuerzo. Por ello, este trabajo tiene por objeto analizar el proceso de evaluación de un edificio escolar particular ubicado en una zona de alta peligrosidad sísmica de Argentina, y el diseño de las estrategias para su refuerzo, según prescripciones del reglamento nacional y americano. Luego de la introducción se describen las características del edificio seleccionado, y se detalla el procedimiento de evaluación seguido, así como los resultados obtenidos en esta etapa. Se discuten también las especificaciones que al respecto se plantean en los reglamentos de los dos países seleccionados. Los resultados de esta evaluación sirven de base para diseñar estrategias de refuerzo, que también son explicadas en el trabajo. A la luz del ejemplo considerado, y de las prescripciones reglamentarias analizadas, se destacan en las conclusiones las partes del proceso evaluación - refuerzo, y se plantea la necesidad de actualizar las recomendaciones en el reglamento argentino.

Palabras claves: vulnerabilidad sísmica, edificios escolares, evaluación y refuerzo.

\section{Introducción}

Los nuevos conocimientos de la ingeniería sismorresistentes no están considerados en construcciones diseñadas y construidas con reglamentos antiguos. Por lo tanto, ante un evento sísmico importante estas construcciones podrían afectar a las personas que se encuentran en ellas. Originándose también daños materiales en la propia construcción, con posible alta demanda de costos y de tiempo para su reparación.

Para estas construcciones en necesario implementar procedimientos de evaluación y posibles refuerzos. Esta temática sigue en la actualidad teniendo importancia, tal como lo muestran los trabajos de FEMA P-2090 (2021) y Razo Carrasco y García Domínguez (2020).

Dentro del grupo de construcciones consideradas, tiene una importancia especial a nivel internacional las instalaciones educativas, tal como se observa en la iniciativa mundial de escuelas seguras (www.wcdrr.org/safeschools.html), que cuenta con el apoyo de Naciones Unidas, UNESCO, UNICEF, etc.

Atento a esta necesidad, este trabajo tiene por objeto analizar el protocolo de evaluación y refuerzo implementado en una escuela de Argentina, ubicada en una zona de alta peligrosidad sísmica, a la luz de las especificaciones de un reglamento nacional y extranjero.

Las características del edificio estudiado en este trabajo se presentan en la sección 2, donde también se indica la razón que motivó la necesidad de la evaluación. El proceso de evaluación llevado a cabo (inspección visual, caracterización de los materiales, relevamiento de patologías y deficiencias, y simulación computacional) se 
describe en la sección 3; comparando también las especificaciones que para este proceso se considera en la norma argentina y americana. Este proceso es la base para el diseño las estrategias de diseño que se presentan en la sección 4, donde se hace referencia también a los requerimientos que se consideran en las normas de los dos países mencionados. El trabajo cierra con las conclusiones (sección 5), donde se destacan las ventajas y limitaciones del proceso evaluación - refuerzo implementado, y se sugieren actualizaciones para lograr que ese proceso, adaptado a edificaciones de Argentina, incorpore los conocimientos actuales.

\section{Consideraciones generales del edificio en estudio}

El edificio escolar, cuya fachada y vista interior se muestra en la Figura 1, está ubicado en la provincia de Mendoza, Argentina, en una zona de mayor peligrosidad sísmica del país. Fue construido en 1983, bajo prescripciones del primer reglamento sismorresistente de la provincia (CCA, 1970).

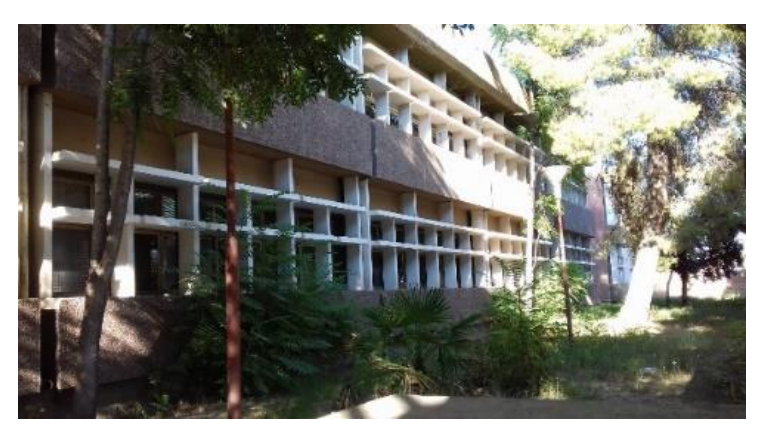

a - Fachada

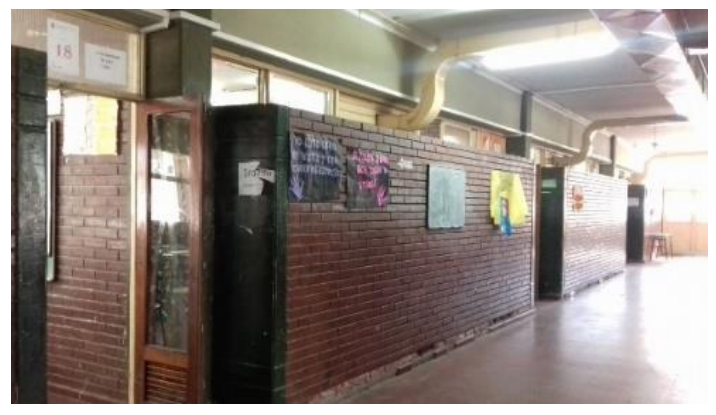

b - Vista pasillo interno

Figure 1 - Edificio escolar en estudio

La construcción, cuyo plano de estructuras se presenta en la Figura 2, consta de 3 bloque (con junta de separación de 3,0 cm): (i) dos laterales de $36.2 \mathrm{~m}$ de longitud y $19.7 \mathrm{~m}$ de ancho, en dos pisos de 3,50 $\mathrm{m}$ de alto cada uno, destinado a aulas; y (ii) un bloque central de 29,10 m de longitud y $17 \mathrm{~m}$ de ancho, también en dos pisos de 3,50 $\mathrm{m}$ de alto cada uno, con destino de oficinas administrativas y aulas (que linda con un patio cerrado con cubierta metálica que también se observa en la Figura 2).

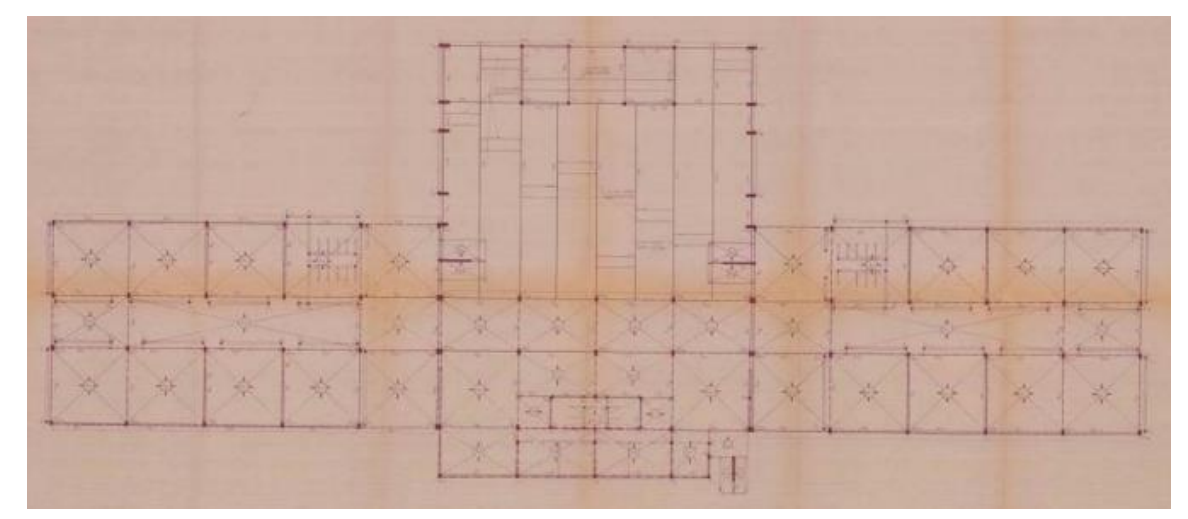

Figure 2 - Plano de estructura del edificio en estudio

La estructura sismorresistente está conformada por losas de hormigón armado, muros de mampostería (con ladrillos macizos en algunos sectores y huecos en otros), pórticos de hormigón armado y el sistema de fundación (vigas y bases aisladas). Según estudio de suelos la construcción se asienta en un sitio tipo $3 \mathrm{~S}_{\mathrm{E}}$, correspondiente a un suelo cohesivo blando de baja plasticidad (INPRES - CIRSOC $103-1,2018$ ), con una velocidad de onda de corte Vs30 $=191 \mathrm{~m} / \mathrm{s}$.

La necesidad de la evaluación fue demandada por la comunidad educativa, al observar fisuras en algunos muros, tal como las que se observan en la Figura 3. 


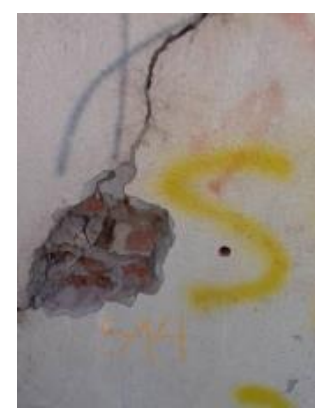

Figure 3 - Una de las fisuras observadas en algunos muros de mampostería.

\section{La evaluación del edificio}

El procedimiento de evaluación permite conocer las características de la estructura y el suelo de fundación en su estado actual, las deficiencias y patologías que la misma presenta, así como la relación demanda vs. suministro según prescripciones reglamentarias (en relación con la resistencia y los desplazamientos).

En las secciones siguientes se sintetizan las condiciones que para la evaluación de estructuras se encuentran en el reglamento argentino y americano, se describe el protocolo de evaluación implementado en la escuela en estudio, y se detalla los resultados obtenidos.

\subsection{Requerimientos reglamentarios}

En el reglamento argentino (INPRES - CIRSOC 103 - I, 2018) tiene un capítulo sobre construcciones existentes, aplicable cuando en ellas se realicen ampliaciones, reformas, o consolidaciones.

Esta norma no especifica un procedimiento de evaluación detallado. Se indica que debe calcularse un índice de seguridad de la construcción ( $r$ ), obtenido como la relación entre la capacidad nominal y la demanda a corte en la base de la construcción. Según el r obtenido la seguridad de la construcción es clasificada como S1 - suficiente ( $r$ igual o mayor 100), S2 - baja ( $r$ entre 80 y 100), S3 - límite ( $r$ entre 60 y 80), y S4 - insuficiente (con $r$ menor a 60).

También debe clasificarse la obra según: (i) su calidad: buena (C1), intermedia (C2), baja (C3) y mala (C4); y (ii) su importancia: importantes (I1), de mediana importancia (12), o de poca importancia (I3).

Luego, para cada calidad e importancia se establece el valor de $r$ que debe alcanzarse con las modificaciones a introducir en la construcción existente. Por ejemplo, para construcciones de calidad intermedia e importantes, se indica que la obra nueva debe alcanzar $100 \%$ de la seguridad de este Reglamento, considerando la contribución de la obra subsistente.

El ACI CODE 318, 2019 tiene un capítulo para considerar la evaluación de la resistencia en estructuras existente, a efectos de verificar si la misma cumple con los requerimientos del código (pudiendo realizar una evaluación de la estructura en forma analítica o experimental). También el ACl cuenta con guías para la inspección visual de estructuras (ACI PRC-220.1, 2008, ACI PRC-364.1, 2019 y ACI CODE 562, 2019).

Un protocolo detallado de evaluación de estructuras, con 3 niveles de profundidad, se encuentra en la norma americana ASCE/SEI 41 (2017), dedicada específicamente a edificaciones existente. La selección de un nivel de evaluación determinado dependerá del objetivo de desempeño que debe alcanzar la construcción para distintos niveles de sismicidad, y del nivel de deficiencias hallado en la evaluación efectuada.

\subsection{Inspección visual}

La inspección visual en el edificio escolar la realizó un equipo de 4 ingenieros, siguiendo los procedimientos de las guías del $\mathrm{ACl}$ antes mencionada. En esta inspección se analizó la documentación existente, se relevaron tipologías estructuras (incluyendo excavaciones para observar las fundaciones), el estado general de la construcción, y las deficiencias detectadas a simple vista. 


\subsection{Caracterización de los materiales que componen la estructura}

Para caracterizar el hormigón se extrajeron testigos, y se realizaron evaluaciones con esclerometría, resistencia a tracción superficial (pull off), y ultrasonido. Mediante un detector de armadura se hicieron verificaciones respecto de la información provista en los planos respectivos. En la Figura 4 se muestras estas evaluaciones.

Los parámetros de los muros de mampostería se tomaron de los valores recomendados por tipo de mampuesto y mortero de asiento del (INPRES - CIRSOC 103 - III, 2018); ya que no se contó en obra con gatos específicos para medir sus propiedades.

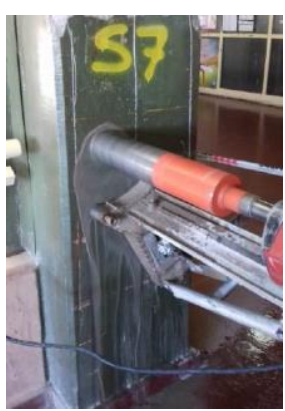

a - Testigos

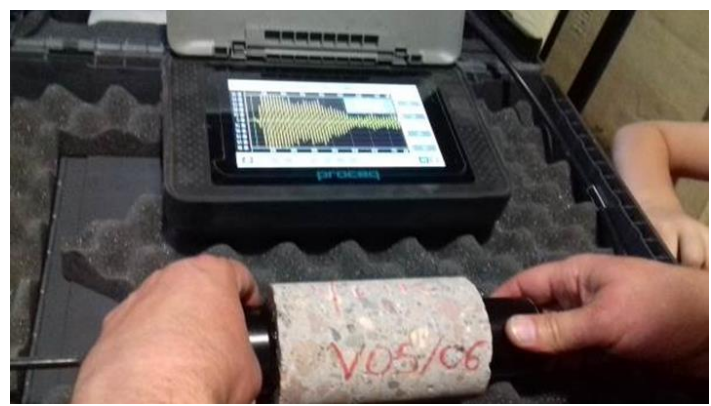

d - Ultrasonido

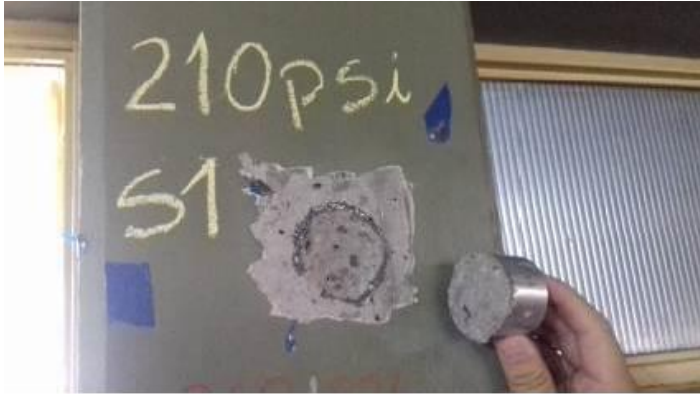

c-Pull off

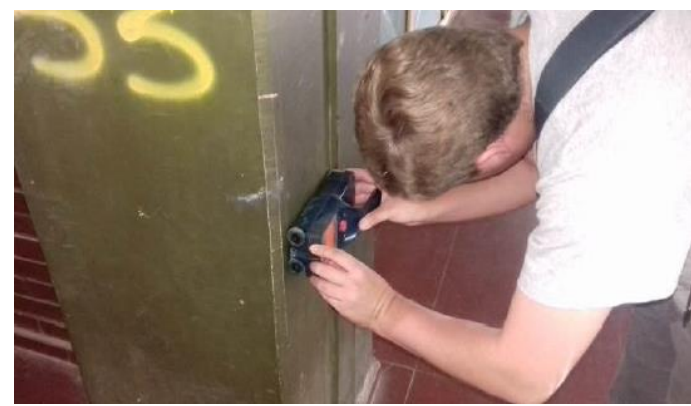

e - Detección de armadura

Figure 4 - Evaluaciones realizadas para caracterizar el hormigón y la armadura

\subsection{Relevamiento de patologías y deficiencias}

Con la extracción de testigos se evaluó el nivel de carbonatación del hormigón, y se observó el estado de la armadura y su recubrimiento, tal como se aprecia en la Figura 5.

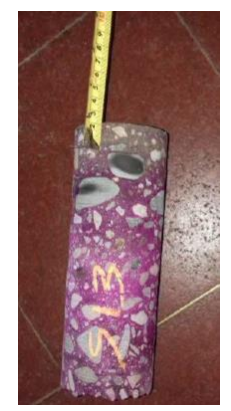

a - Carbonatación

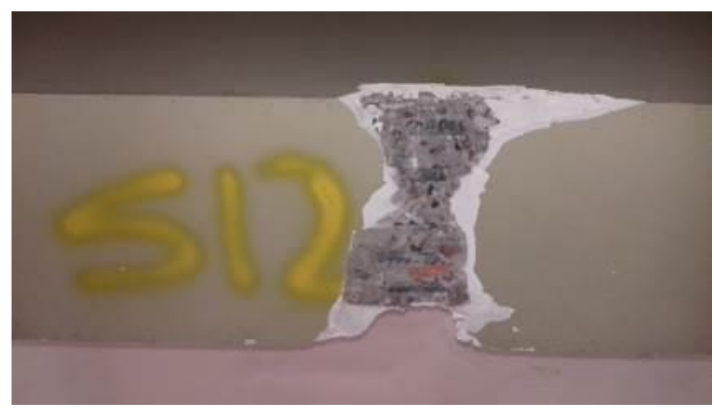

b - Visualización del estado de la armadura

Figure 5 - Evaluaciones realizadas para detectar patologías 


\subsection{Simulación computacional de la estructura en su estado actual}

Con la información obtenida en las etapas anteriores se ejecutó un modelo estructural de cada bloque del edificio, en un programa computacional de elementos finitos. Estos modelos fueron analizados por el método estático y por el método de análisis dinámicos lineal en el tiempo (ya que los análisis no lineales no son considerados en el reglamento argentino), en base a 3 registros sísmicos escalados según el espectro de diseño del reglamento argentino (INPRES - CIRSOC 103 -I, 2018).

El modelo permitió determinar el índice $r$ de seguridad de la construcción, y las derivas de piso.

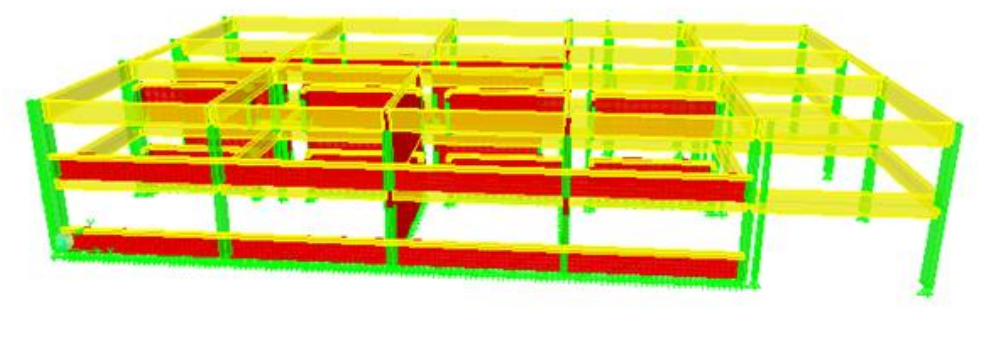

Figure 6 - Modelo computacional del edificio escolar (bloque lateral)

\subsection{Informe de evaluación}

Como resultado de esta evaluación se logró verificar las tipologías estructurales respecto a la información provista en planos existentes, las características del sitio, y establecer la resistencia característica del hormigón y la armadura.

Se detectaron: (i) deficiencias: existencia de columnas cortas, empleo de ladrillos huecos, asentamiento en zonas cercanas a baños, existencia de jardines colindantes a la construcción, patios con desagües hacia los bloques; y (ii) patologías: fisuras en muros y en cimentaciones (como se muestra en Figura 7), y filtraciones en techos. Se observó además que el frente de carbonatación no había alcanzado a la armadura, y no se detectó corrosión en ella.

Del análisis computacional se determinó un índice $r$ menor a 100 (capacidad insuficiente del suelo de fundación, de muros de mampostería, y de columnas y vigas); y desplazamientos horizontales que indican la posibilidad de choque entre los distintos bloques.

Se concluye entonces que es necesario implementar estrategias de refuerzo (para lograr un índice de $r$ igual o mayor a 100 y controlar las derivas de piso), y salvar las deficiencias y patologías relevadas.

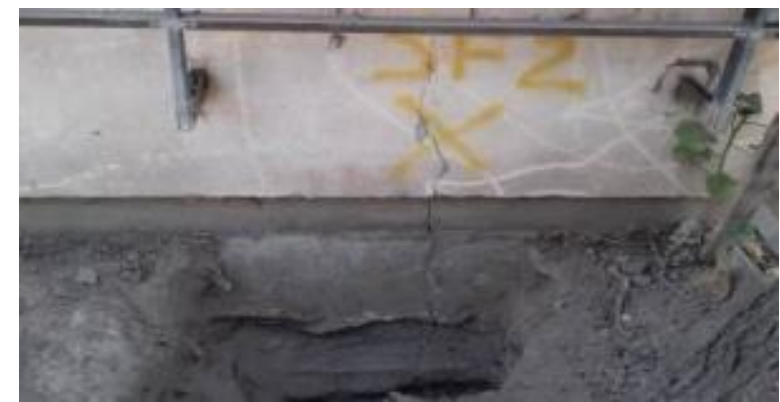

Figure 7 - Existencia de fisuras en el sistema de fundación 


\section{El refuerzo del edificio}

Las estrategias de refuerzo de una construcción deben atender las deficiencias y patologías halladas durante el proceso de evaluación. Deben considerar también la factibilidad técnica y económica de su implementación en el lugar donde la obra se encuentra.

En el diseño de estas estrategias habrá que seguir especificaciones reglamentarias, por lo que en esta sección se comparan primero los requisitos generales que para el refuerzo de estructuras establecen el reglamento argentino y americano.

También se describe los refuerzos que se diseñaron para la escuela en estudio, y se analiza una propuesta de refuerzo que considera los sistemas de disipación pasiva de energía.

\subsection{Requerimientos reglamentarios}

El capítulo del reglamento argentino (INPRES - CIRSOC 103 - I, 2018) alusivo a construcciones existente no hace referencias a las posibles estrategias refuerzo a implementar en estructuras existentes. Solo establece las exigencias y comprobaciones que deben realizarse para la construcción modificada por el refuerzo propuesto. Indica que debe alcanzarse en el edificio reforzado un nivel de seguridad según sea la calidad sismorresistente de la obra primitiva y la importancia de la obra actual. Cabe aclarar que este reglamento considera un solo nivel de demanda sísmica (dado por el espectro de diseño) para el cuál se espera el nivel de desempeño de seguridad de vida.

La norma americana específica para construcciones existentes (ASCE/SEI 41, 2017) establece requerimientos de refuerzo estructural para los objetivos de desempeño que se fijen para los distintos niveles de sismicidad. Establece también requerimientos especiales para las fundaciones y para estructuras de acero, hormigón armado y mampostería. Considera también como estrategias de refuerzo a los sistemas de aislamiento y los sistemas de disipación pasiva de energía.

\subsection{Estrategias de refuerzo implementadas}

Las estrategias de refuerzo propuesta para un edificio escolar deben considerar los costos de su ejecución, los tiempos que demanda su implementación (con la posibilidad de que el edificio pueda seguir en uso en algunos sectores), y las tecnologías que estén disponibles en la región donde se ubique la misma (considerando también la mano de obra disponible para realizar ese trabajo).

Para la escuela en estudio, y en función del resultado del proceso de evaluación y de las consideraciones recién mencionadas, se propuso: (i) refuerzo del sistema de fundación (agregando elementos estructurales y recreciendo los existentes); (ii) refuerzo de columnas y vigas con FRP (también para las columnas cortas); (iii) refuerzo de muros de mampostería con el agregado de mallas de armadura y hormigón proyectado; (iv) aumento de la rigidez a desplazamiento horizontales con la incorporación de riostras metálicas; (v) fijación de elementos no estructurales; y (vi) modificaciones necesarias para salvar las deficiencias y patologías consideradas durante la evaluación del edificio. En la Figura 8 pueden visualizarse alguna de las estrategias de refuerzo propuestas.

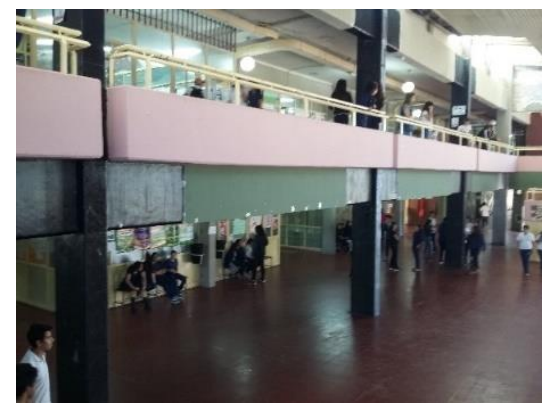

a - Refuerzo de columnas y vigas

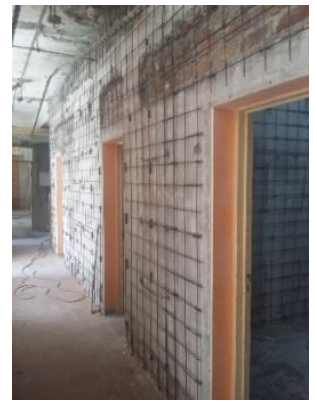

b - Refuerzo de muros

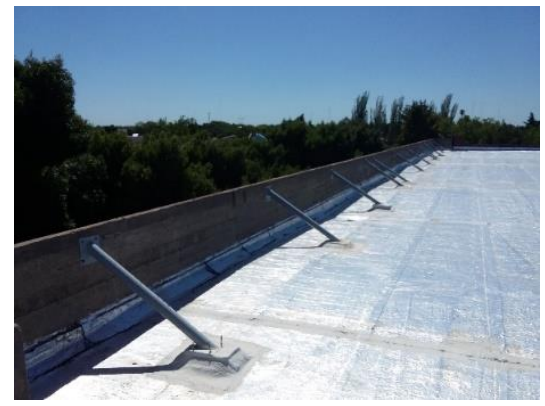

c - Refuerzo de elementos no estructurales

Figure 8 - Ejemplos de estrategias de refuerzo diseñadas para el edificio en estudio 


\subsection{Otra estrategia de refuerzo considerada}

Dado que entre las estrategias de refuerzo mencionadas en la sección anterior se consideró el uso de riostras metálicas, también se evaluó para los bloques laterales el reemplazo de las mismas por un sistema de disipación pasiva de energía, consistente en riostras que no pandean, y denominadas barras de pandeo restringido.

Para esta estrategia, y considerando los requerimientos de la norma americana (ASCE/SEI 41,2017) se realizó un análisis dinámico no lineal en base a 14 registros símicos escalados según el sismo de diseño del reglamento argentino (INPRES - CIRSOC 103 - I, 2018), y una propuesta de sismo máximo considerado (no contemplado en el reglamento mencionado).

En la Figura 9 se muestran la disposición de disipadores de energía (elementos K1 a K12) propuestos para el refuerzo de los pórticos longitudinales en los bloques laterales. Mientras que en la Figura 10 se observan espectros de los registros escalados al sismo de diseño del reglamento argentino, y la deriva de piso para la demanda del sismo de diseño (SD) y el máximo esperado (MCER), bajo 7 registros de falla lejana (FF) y otros 7 de falla cercana (NF). Más detalles de este estudio se tiene en Palazzo et al. (2021).

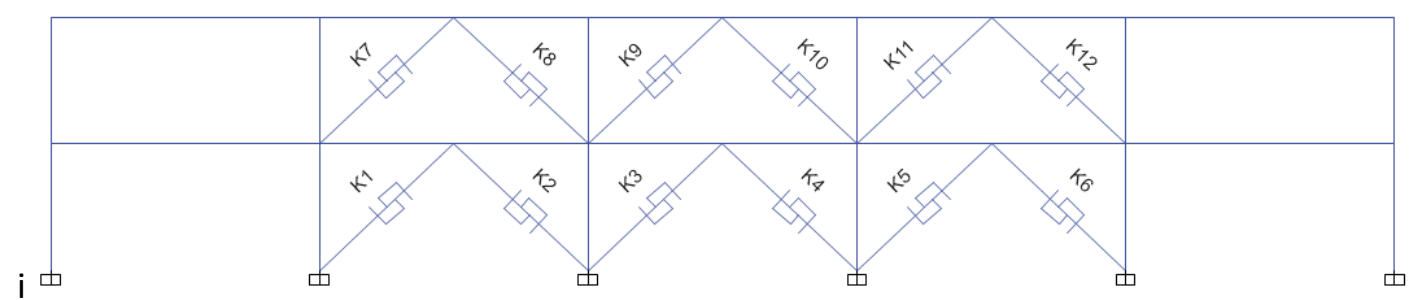

Figure 9 - Disposición de barras de pandeo restringido en los pórticos de los bloques laterales

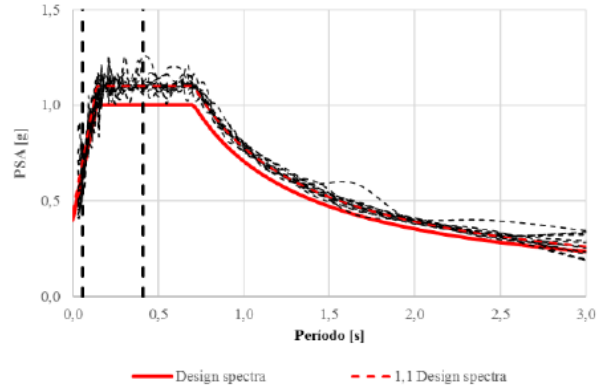

a - Registros escalados

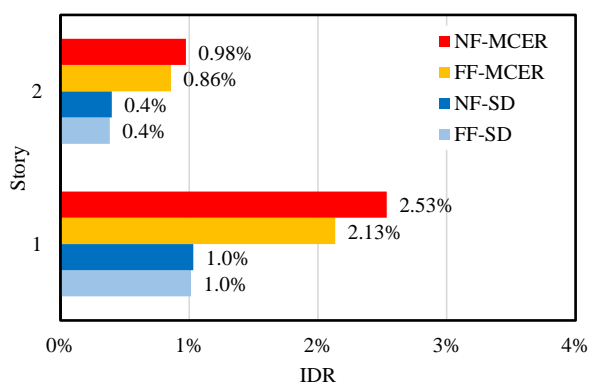

b - Derivas de piso

Figure 10 - Demanda y respuesta del pórtico con sistema de disipación pasiva de energía

\section{Conclusiones}

Las construcciones existentes pueden ser vulnerables ante un evento sísmico; siendo necesario su evaluación y posible refuerzo. Es fundamental entonces contar con un procedimiento de evaluación que permita primero conocer todas las deficiencias y patologías que se hayan presentes en el edificio estudiado. Luego habrá que analizar estrategias de refuerzo que permitan subsanar los problemas encontrados, considerando también la factibilidad técnica y económica de su implementación.

Si entre las construcciones existentes consideramos a los edificios escolares, estos presentan especial importancia por el uso a los que están destinados. Sería conveniente que a nivel social y gubernamental se especifiquen objetivos de desempeño para estas construcciones. Los autores consideran conveniente fijar al menos un nivel de desempeño para el sismo de diseño, y otro para el máximo considerado. 
Los reglamentos deberían proponer procedimientos de evaluación y posibles estrategias de refuerzo que hayan demostrado su eficiencia. También deberían especificar criterios de aceptación de la respuesta bajo los dos niveles de demanda sísmica mencionados, para cada elemento estructural y para la construcción en su conjunto. Así, por ejemplo, en el caso de pórticos de hormigón armado estas verificaciones podrían establecer valores máximos de la respuesta en rotación plástica y en derivas de piso.

En este trabajo, y para un edificio escolar de Argentina ubicado en una zona de máxima peligrosidad sísmica, se ha detallado el proceso implementado en la práctica para su evaluación y el refuerzo. También se ha considerado como posible estrategia de refuerzo a una de las nuevas tecnologías, como son los sistemas de disipación pasiva de energía. Se ha seguido en general las especificaciones que para construcciones existentes se establecen en el reglamento argentino, las cuales han sido complementadas por guías y normas americanas.

A la luz de los conocimientos actuales parece que el reglamento argentino necesita una actualización en lo referente a estructuras existentes. Los documentos americanos considerados en este trabajo presentan gran cantidad de información que podrían servir de base para que el reglamento de Argentina proponga metodologías de evaluación y refuerzo consistente con el contexto de las construcciones de nuestro país.

La profundización del camino desde la evaluación hacia el refuerzo, adaptado a cada país, es lo que permitirá arribar a construcciones menos vulnerables.

\section{Agradecimientos}

Los autores agradecen a la Universidad Tecnológica Nacional por el financiamiento del proyecto relativo a este estudio, y a SOLIDUS - Ingenieros Consultores (www.solidus-ing.com) por la información facilitada.

\section{Referencias}

ACI CODE 318 (2019). Building Code Requirements for Structural Concrete and Commentary. American Concrete Institute.

ACI CODE 562 (2019). Code Requirements for Assessment, Repair, and Rehabilitation of Existing Concrete Structures and Commentary. American Concrete Institute.

ACI PRC-220.1 (2008). Guide for Conducting a Visual Inspection of Concrete in Service. American Concrete Institute.

ACI PRC-364.1 (2019). Guide for Assessment of Concrete Structures Before Rehabilitation. American Concrete Institute.

ASCE/SEI 41 (2017). Seismic evaluation and retrofit of existing buildings. American Society of Civil Engineers.

CCA (1970). Código de Construcciones Antisísmicas. Provincia de Mendoza.

FEMA P-2090 (2021). Recommended options for improving the built environment for post-earthquake reoccupancy and functional recovery time. Federal Emergency Management Agency and National Institute of Standards and Technology, https://doi.org/10.6028/NIST.SP.1254.

INPRES - CIRSOC 103 - I (2018). Reglamento argentino para construcciones sismorresistentes. Parte I Construcciones en general. INPRES e INTI - CIRSOC.

INPRES - CIRSOC 103 - III (2018). Reglamento argentino para construcciones sismorresistentes. Parte III Construcciones de mampostería. INPRES e INTI - CIRSOC.

Palazzo, G., Bay, C., Prados, J., Vielma, J. and Calderon, F. (2021). Performance assessment of scholar building retrofitted with passive energy dissipation system. 17th World Conference on Earthquake Engineering, Sendai - Japan.

Razo Carrasco, D. y García Domínguez, O. (2020). Evaluación integral de la seguridad estructural de edificaciones existentes dañadas por sismos de gran magnitud. Revista de Ingeniería Sísmica No. 104, 5171. 